\title{
Measuring cosmological distances by coalescing binaries
}

\author{
I. De Martino*, S. Capozziello ${ }^{\dagger}$, M. De Laurentis ${ }^{\dagger}$ and M. Formisano** \\ *Física Teórica, Universidad de Salamanca, 37008 Salamanca, Spain \\ †Dipartimento di Scienze Fisiche, Università di Napoli "Federico II" and INFN sez. di Napoli \\ Compl. Univ. di Monte S. Angelo, Edificio G, Via Cinthia, I-80126 - Napoli, Italy \\ ** Dipartimento di Fisica, Università di Roma "La Sapienza", Piazzale Aldo Moro 5, I-00185 \\ Roma, Italy and INAF-IAPS, Via Fosso del Cavaliere 100, 00133 Roma (Italy)
}

\begin{abstract}
Gravitational waves detected from well-localized inspiraling binaries would allow us to determine, directly and independently, binary luminosity and redshift. In this case, such systems could behave as "standard candles" providing an excellent probe of cosmic distances up to $z<0.1$ and complementing other indicators of cosmological distance ladder.
\end{abstract}

Keywords: Gravitational waves, standard candles, cosmological distances.

PACS: 04.25.dg, 04.25.dk, 04.30.-w, 98.80.Es

\section{INTRODUCTION}

One of the fundamental goals of the modern Cosmology is to measure the distance of the emitting systems, a key parameter linked to the size, energy and mass of them. In particular, extragalactic distance measurements are fundamental in the learning of the size, age, composition and evolution of the Universe [1,2]. Depending on the distance of the object, the method of the measure changes radically. There are several problems in the cosmic distance ladder due to this approach, for which we can not discriminate the best parametrization (in term of dark energy and dark matter) [2]. Among these, the main one is that the errors and the uncertainties increase at each steps. Another one is linked to the calibration: in fact, we have to determinate a class of object with absolute magnitude very well-known. We could resolve a part of these problems using another type of distance indicator for which we don not use the electromagnetic flux but the gravitational one. Here we show that the coalescing binary systems could be used to determine independently the distances, so we can reduce the errors and the uncertainties and we can improve the calibration.

\section{COALESCING BINARIES SYSTEM AS STANDARD CANDLES}

A binary system is composed of two stars (neutron stars (NS), white dwarves (WD) and black holes $(\mathrm{BH})$ ) orbiting around their center of mass, whose components inspiral and loss angular momentum and energy via gravitational waves (GWs) emission. As a consequence of the coalescence GWs frequency increases and, if it is observed, it could be a "signature" for these dynamic systems. For this reason they are usually considered 
strong emitter of GWs and they could be extremely useful for cosmological distance ladder if the physical features of GWs emission are well determined. For the coalescing systems, we can define:

$$
M_{c}=\frac{\left(m_{1} m_{2}\right)^{3 / 5}}{\left(m_{1}+m_{2}\right)^{1 / 5}},
$$

a particular combination of the masses of the two stars, known as the chirp mass. By determining the amplitudes for the two polarizations of the GWs at lowest order in $v / c$ [6], and by averaging over the orbital period and the orientation of the binary orbital plane, we can write the average (characteristic) GWs amplitude:

$$
h_{c}(t)=\frac{4}{r(z)}\left(\frac{G \mathscr{M}_{c}(z)}{c^{2}}\right)^{5 / 3}\left(\frac{\pi f(t)}{c}\right)^{2 / 3} .
$$

Eq. (2) has to be modified if we consider a binary at a cosmological distance, i.e. at redshift $z$. By considering the propagation of the GWs in a Friedmann-Robertson-Walker Universe and by taking into account that all quantities appearing in Eq. (2) are measured by the observer, we have simply to apply the redshift correction (due to expansion of the Universe), as follows:

1. The frequency $f(t)$ is replaced by $f_{\text {obs }}$, which is the frequency measured by the observer. It is redshifted with respect to the source frequency $f_{s}$, i.e. $f_{\text {obs }}=f_{s} /(1+$ $z)$;

2. the chirp mass $M_{c}$ must be replaced by $\mathscr{M}_{c}=(1+z) M_{c}$;

3 . the distance $r$ to the source must be replaced by the luminosity distance $d_{L}(z)$.

If we are able to measure the quantities related to GWs emission $\left(h_{c}(t), f(t)\right)$ and if we can estimate the chirp mass and the redshift $(z)$ then we can determine the cosmological distance $d_{L}$, founding a gravitational standard candle and testing the cosmological model [7]. For the determination of the redshift $z$, several possibilities have been proposed, like for example the searching of an optical counterpart. In particular, short GRBs appear related to such systems and they are quite promising potential GWs standard sirens $[8,9]$. Another possibility, adopted in this work, is that the redshift of the binary systems can be associated to the barycenter of the galaxy or the galaxy cluster hosting the systems.

\section{NUMERICAL ANALYSIS AND RESULTS}

We consider coalescing binary systems at redshifts (without uncertainties) $z<0.1$ without any systematic errors in order to obviate the absence of a complete catalogue of such systems. The choice of low redshifts is due to the observational limits of groundbased interferometers like VIRGO or LIGO $[4,3]$. In the simulation here presented, the sources are slightly out of LIGO-VIRGO observable range, but in principle, they are within future improvements like Advanced VIRGO and Advanced LIGO.

Here, we have used the redshifts taken by NASA/IPAC EXTRAGALACTIC DATABASE (NED) [10], fixing them at the barycenter of the host galaxy/cluster. 
TABLE 1. For each clusters we indicate redshifts, characteristic amplitudes, frequency range for coalescing binary systems.

\begin{tabular}{|c|c|c|c|}
\hline Object & $\mathbf{z}$ & $h_{c}$ & $\begin{array}{c}\text { Freq. } \\
(\mathrm{Hz})\end{array}$ \\
\hline NGC 5128 & 0.0011 & $10^{-22}$ & $0 \div 10$ \\
NGC 1023 Gr. & 0.0015 & $10^{-22}$ & $0 \div 10$ \\
NGC 2997 & 0.0018 & $10^{-22}$ & $5 \div 15$ \\
NGC 5457 & 0.0019 & $10^{-22}$ & $10 \div 20$ \\
NGC 5033 & 0.0037 & $10^{-22}$ & $25 \div 35$ \\
Virgo Cl. & 0.0042 & $10^{-22}$ & $30 \div 40$ \\
Fornax Cl. & 0.0044 & $10^{-22}$ & $35 \div 45$ \\
NGC 7582 & 0.0050 & $10^{-22}$ & $45 \div 55$ \\
Ursa Major Gr. & 0.0057 & $10^{-22}$ & $50 \div 60$ \\
Eridanus Cl. & 0.0066 & $10^{-22}$ & $55 \div 65$ \\
\hline
\end{tabular}

(a) NS-NS systems.

\begin{tabular}{|c|c|c|c|}
\hline Object & $\mathbf{z}$ & $h_{c}$ & $\begin{array}{c}\text { Freq. } \\
(\mathrm{Hz})\end{array}$ \\
\hline NGC 5128 & 0.0011 & $10^{-22}$ & $0 \div 10$ \\
NGC 1023 Gr. & 0.0015 & $10^{-22}$ & $0 \div 10$ \\
NGC 2997 & 0.0018 & $10^{-22}$ & $5 \div 15$ \\
NGC 5457 & 0.0019 & $10^{-22}$ & $10 \div 20$ \\
NGC 5033 & 0.0037 & $10^{-22}$ & $25 \div 35$ \\
Virgo Cl. & 0.0042 & $10^{-22}$ & $30 \div 40$ \\
Fornax Cl. & 0.0044 & $10^{-22}$ & $35 \div 45$ \\
NGC 7582 & 0.0050 & $10^{-22}$ & $45 \div 55$ \\
Ursa Major Gr. & 0.0057 & $10^{-22}$ & $50 \div 60$ \\
Eridanus Cl. & 0.0066 & $10^{-22}$ & $55 \div 65$ \\
\hline
\end{tabular}

(b) BH-BH systems.

The binary chirp mass $M_{C}$ is typically measured from the newtonian part of the signal at upward frequency sweep, to $\sim 0.04 \%$ for a NS/NS binary and $\sim 0.3 \%$ for a system containing at least one $\mathrm{BH}[5,11]$. The luminosity distance of the binary system can be inferred, from the observed waveforms, to a precision $\sim 3 / \rho \leq 30 \%$, where $\rho=S / N$ is the amplitude signal-to-noise ratio in the total LIGO network. By fixing the characteristic amplitude of GWs, we tune the frequencies in a range compatible with such a fixed amplitude, then the error on distance luminosity is calculated by the error on the chirp mass with standard errors propagation.

The systems here considered are NS-NS and BH-BH. For each of them, a particular frequency range and a characteristic amplitude (beside the chirp mass) are fixed. We start with the analysis of NS-NS systems $\left(M_{C}=1.22 M_{\odot}\right)$ with characteristic amplitude fixed to the value $10^{-22}$. In Table 1(a), we report the redshift, the value of $h_{C}$ and the frequency range of systems analyzed. In Fig. 1(a), the derived Hubble relation is reported. The Hubble constant value is $72 \pm 1 \mathrm{~km} / \mathrm{s} \mathrm{Mpc}$ in agreement with the recent WMAP estimation [13]. The same procedure is adopted for $\mathrm{BH}-\mathrm{BH}$ systems $\left(M_{C}=8.67 M_{\odot}, h_{C}=10^{-21}\right)$. The data and the results are respectively in Table 1(b) and in Fig. 1(b), and the Hubble constant value computed by these systems is $69 \pm 2$ $\mathrm{km} / \mathrm{s} \mathrm{Mpc}$. In this case the error is larger than the previous one, because we have a larger indetermination in the measure of the mass of the $\mathrm{BH}$.

\section{CONCLUSIONS}

We have considered simulated binary systems, whose redshifts can be estimated considering the barycenter of the host astrophysical system as galaxy, group of galaxies or cluster of galaxies. We have simulated NS-NS and BH-BH binary systems. Clearly, the leading parameter is the chirp mass $M_{c}$, or its red-shifted counter-part $\mathscr{M}_{c}$, which is di- 


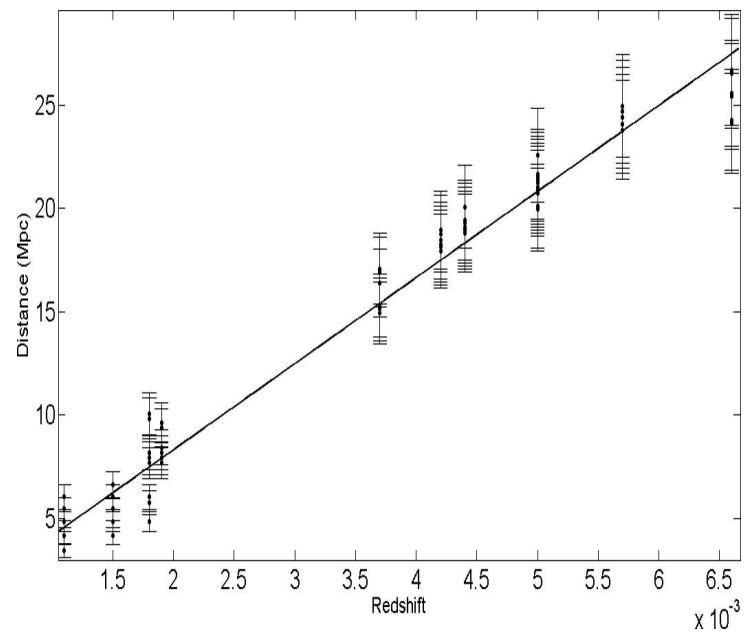

(a) NS-NS systems.

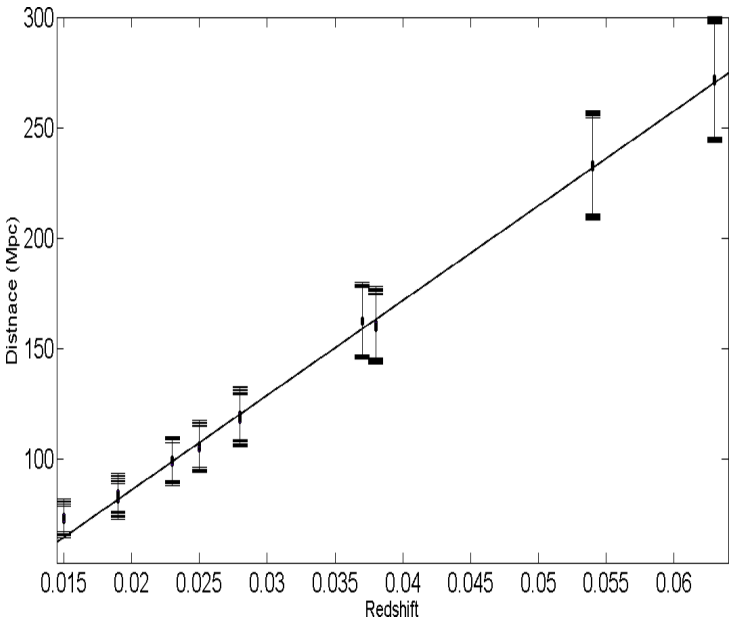

(b) $\mathrm{BH}-\mathrm{BH}$ systems.

FIGURE 1. Luminosity distance vs redshift for simulated systems.

rectly related to the GWs amplitude. The adopted redshifts are in a well-tested range of scales and the Hubble constant value is in good agreement with WMAP estimation. The Hubble-luminosity-distance diagrams of the above simulations show the possibility to use the coalescing binary systems as distance indicators. The limits of the method are, essentially, the measure of GWs polarizations and the redshifts but it could lead to completely independent determinations and it could complement and increase the confidence of other standard candles [12].

\section{REFERENCES}

1. Freedman, W.L. and Madore, B.F., 2010, ARA\&A, 48: 673-710.

2. Frieman et al., 2008, ARA\&A, 46, 385-432.

3. Abramovici A., et al., 1992 Science 256, 325; http://www.ligo.org

4. Caron B. et al., 1997 Class. Quant. Grav. 14, 1461.http://www.virgo.infn.it

5. Capozziello S., De Laurentis M., De Martino I., Formisano M., 2010 Astropart. Physics 33, 190.

6. Maggiore M., 2007 Gravitational Waves, Volume 1: Theory and Experiments, Oxford Univ. Press

7. Schutz B.F., 1986, Nature, 323, 310.

8. Dalal et al., 2006, PRD, 74, 063006.

9. Capozziello S., De Laurentis M., De Martino I., Formisano M., 2010, Astroph. and Sp. Sc., $332,35$.

10. Abell, G., Corwin, H., Olowin R., 1989, Astrophys. J. Suppl., 70, 1.

11. Cutler C. and Flanagan E.E., 1994, PRD, 49, 2658.

12. Holz, D.E., Hughes, S.A., 2005, Astrophys.J. 629, 15.

13. Larson D., et.al., 2011, Astrophys.J.S., 192, 16. 